\title{
Multi-Target Localization in Asynchronous MIMO Radars Using Sparse Sensing
}

\author{
Saeid Sedighi, Bhavani Shankar Mysore R, Sina Maleki, and Björn Ottersten \\ SnT, University of Luxembourg, Luxembourg city, Luxembourg \\ E-mails: \{saeid.sedighi,bhavani.shankar,sina.maleki,bjorn.ottersten\}@ uni.lu
}

\begin{abstract}
Multi-target localization, warranted in emerging applications like autonomous driving, requires targets to be perfectly detected in the distributed nodes with accurate range measurements. This implies that high range resolution is crucial in distributed localization in the considered scenario. This work proposes a new framework for multi-target localization, addressing the demand for the high range resolution in automotive applications without increasing the required bandwidth. In particular, it employs sparse stepped frequency waveform and infers the target ranges by exploiting sparsity in target scene. The range measurements are then sent to a fusion center where direction of arrival estimation is undertaken. Numerical results illustrate the impact of range resolution on multi-target localization and the performance improvement arising from the proposed algorithm in such scenarios.
\end{abstract}

Index Terms-localization, stepped frequency modulation, joint range-DoA estimation, sparse sensing, $\ell_{1}$ optimization.

\section{INTRODUCTION}

Target localization is one of the fundamental tasks of Multiple Input Multiple Output (MIMO) radar systems and it has received a considerable attention in the last few years [1]-[3]. In general, target localization techniques can be categorized under two main approaches with regard to the MIMO radar system architecture. The first approach develops the localization algorithms based on a fully-synchronized collocated MIMO radar system [4], [5]. Although the collocated MIMO techniques are able to achieve high localization performance through providing the waveform diversity [6], they demand a complicated transceiver structure with expensive devices to enable the coherent processing required. However, many commercial applications, such as automotive radar, are required to localize the targets using cheap devices. This capability is enabled by the second approach based on distributed localization techniques where simple, independent radar nodes are used instead of a complicated collocated radar system. Distributed localization techniques exploit the angular (spatial) diversity, provided by spatially separated radar nodes, to estimate targets locations [6].

In addition to lack of support for coherent processing, the independent radar nodes may only provide coarse local Direction of Arrival (DoA) information. This is indeed the case when each of the nodes is equipped with a single or a few antenna elements, as envisaged in automotive applications [7], due to cost and implementation issues. Thus, the radar nodes have access only to fine range, or equivalently time delay, measurements. Several approaches have been proposed in the literature to estimate the targets locations in a distributed manner using range measurements provided by distributed radar nodes [8]-[12]. Best Linear Unbiased Estimates (BLUE) of the target locations have been derived in [8] by linearizing the elliptic equations corresponding to the target positions. A two-stage weighted least square approach is proposed in [11], which is further demonstrated to achieve Cramer-Rao Lower bound (CRLB) in the high Signal-to-Noise-Ratio (SNR) regime.

Evidently, the performance of multi-target distributed localization relies heavily on perfect target detection at each of the nodes. Erroneous target detection can significantly degrade the localization performance regardless of the algorithm used. Further, since the radar nodes are closely located in automotive applications (e.g. on the bumper), small errors in range measurements can affect the localization. Therefore, high range resolution is a must in multi-target distributed localization for automotive application. However, achieving such a high range resolution using conventional methods demands high bandwidths which may not be affordable in practice. Thus, in such applications, the need for developing new algorithms, which are able to provide the desired range resolution without additional requirements on bandwidth, is seriously felt.

Towards achieving enhanced multi-target localization for automotive application, we propose a framework based on Sparse Stepped Frequency Waveforms (SSFW) and sparse sensing. Specifically, exploiting the sparsity in the target scene, the framework uses a two-step decentralized procedure for localization. In the first step, the target detection and super-resolution range estimation are undertaken. The link between range resolution and the bandwidth is broken by proposing a SSFW and then estimating the target ranges using the sparse sensing technique. It is shown that the proposed approach provides super-resolution estimates of the targets ranges enabling multi-target resolution with affordable bandwidths. The waveform design parameters offer additional degrees-of-freedom for efficient sparse sensing. In the second step, the precise range measurements are transmitted to a Fusion Center (FC), where the DoA estimation is performed. The Maximum Likelihood Estimation (MLE) is used in the FC for the DoA estimation and subsequent localization.

Notation: Vectors and matrices are referred to by lower- and upper-case bold-face, respectively. The superscripts $*, T$ and $H$ denote the conjugate, transpose and Hermitian (conjugate transpose) operations, respectively. $\|\mathbf{a}\|_{1}$ and $\|\mathbf{a}\|_{2}$ stand for 


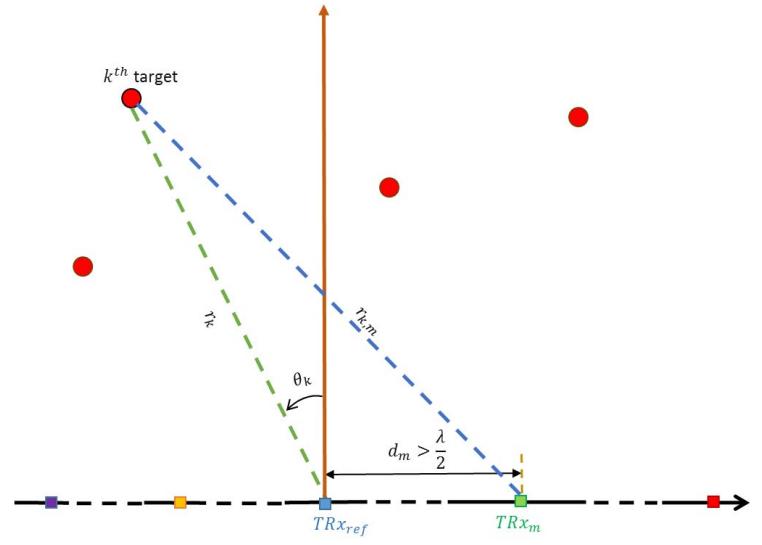

Fig. 1. Distributed radar system model

the $\ell_{1}$ - and $\ell_{2}$-norm of the vector a, respectively. $|\mathcal{A}|$ and $\mathcal{A}(n)$ represent the cardinality and the $n^{\text {th }}$ element of the set $\mathcal{A}$, respectively. $\operatorname{diag}\left\{c_{1}, c_{2}, \cdots, c_{M}\right\}$ is a diagonal matrix with diagonal entries of $c_{1}, c_{2}, \cdots, c_{M}$.

\section{BASIC AsSumptions AND System MODEL}

We consider a distributed radar system (see Fig. 1) including $M$ mono-static radar nodes which are linearly distributed so that the $m^{\text {th }}$ radar node is located at distance $d_{m}$ from some reference point. The reference point can also be one of the radar nodes. The distances of the radar nodes from the reference point is assumed to be greater than half of the operational wavelength. Each radar node is composed of $D$ patch antennas embedded in an integrated chip, which beamform the transmit signal in a particular direction at each sensing time. The transmit beams are designed such that the beams of a subset $\mathcal{A}$ of the set of radar nodes intersect at each sensing time, with $|\mathcal{A}| \geq 2$. No synchronization is assumed between the radar nodes, although the patch antennas in each node are assumed to be synchronous. It is also supposed that $K$ stationary point targets are present in the area covered by the beams of radar nodes within the subset $\mathcal{A}$. The $k^{\text {th }}$ is located at distance $r_{k}$ and azimuth angle $\theta_{k}$ with respect to the reference point. The targets are assumed to be in the far-field, i.e., $r_{k} \gg d_{m}$. Such a system model is valid, for example, in automotive applications where the radar transceivers are installed on the car-bumpers. We note that the data association problem, arising in multi-target scenario, is not considered in this paper. Indeed, it is assumed that the signals backscattered from different targets are properly associated in a preceding processing step.

We employ a SSFW for target illumination by each of the nodes. The SSFW is a variation of Stepped Frequency Waveforms (SFWs). A Classical SFW (CSFW) is composed of $P$ consecutive monotone narrowband pulses with duration $T_{c}$ so that the frequency of the $p^{\text {th }}$ pulse is assumed to be $f_{p}=f_{c}+p \triangle f$, where $p \in \mathcal{P} \doteq\{1,2, \cdots, P\}, f_{c}$ is the carrier frequency and $\triangle f$ denotes the frequency step size. The bandwidth and Coherent Processing Interval (CPI) of the CSFW are $P \triangle f$ and $P T_{c}$, respectively. The SSFW can be made out of the CSFW by transmitting only portions of the monotone narrowband pulses of the CSFW in a CPI. Indeed, the frequency of the $n^{\text {th }}$ pulse of the SSFW is $f_{n}=$ $f_{c}+\mathcal{S}(n) \triangle f$, where $\mathcal{S} \subseteq \mathcal{P}$ with $|\mathcal{S}|=N \ll P$. The subset $\mathcal{S}$ can be selected from the set $\mathcal{P}$ in a deterministic or random fashion so as to achieve a desired inference performance [13]. The bandwidth and CPI of the SSFW are respectively $N \triangle f$ and $N T_{c}$, which are considerably smaller than those of the CSFW.

Without loss of generality, it can be supposed that all transmit nodes use the same carrier frequency ${ }^{1}$. Hence, the $n^{\text {th }}$ pulse transmitted by the $m^{\text {th }}$ radar node, where $m \in \mathcal{A}$, can be modeled as

$T_{m}(n, t)=s_{m}\left(t-n T_{c}\right) \exp \left\{j 2 \pi\left(f_{c}+\mathcal{S}(n) \triangle f\right)\left(t-n T_{c}\right)\right\}$,

where $0 \leq n \leq N-1$, and $s_{m}(t), 0<t<T_{c}$, denotes the complex envelope of the monotone narrowband pulse transmitted by the $m^{\text {th }}$ radar node. The transmitted waveforms are assumed to be almost orthogonal across the $M$ transmit nodes, i.e., $\frac{1}{T_{c}} \int_{0}^{T_{c}} s_{m}(t) s_{m^{\prime}}^{*}(t-\tau) d t, m \neq m^{\prime}$, is assumed to be negligible compared to $\frac{1}{T_{c}} \int_{0}^{T_{c}} s_{m}(t) s_{m}^{*}(t-\tau) d t$. Assuming that the propagation is non-dispersive, the received signal at the location of the $k^{\text {th }}$ target equals

$$
\begin{aligned}
v_{k}(n, t) & =\sum_{m=1}^{M} s_{m}\left(t-n T_{c}-\tau_{m, k}\right) \\
& \times \exp \left\{j 2 \pi\left(f_{c}+\mathcal{S}(n) \triangle f\right)\left(t-n T_{c}-\tau_{k, m}\right)\right\},
\end{aligned}
$$

where $\tau_{k, m}=\frac{r_{k, m}}{c}$ and $c$ denotes the speed of light in vacuum. The backscattered signal from $K$ targets at the $i^{\text {th }}$ radar node, where $m \in \mathcal{A}$, is then described by

$$
\begin{aligned}
y_{i}(n, t)= & \sum_{k=1}^{K} \sum_{m=1}^{M} \alpha_{k} s_{m}\left(t-n T_{c}-\tau_{k, m}-\tau_{k, i}\right) \\
& \times \exp \left\{j 2 \pi\left(f_{c}+\mathcal{S}(n) \triangle f\right)\left(t-n T_{c}-\tau_{k, m}-\tau_{k, i}\right)\right\} \\
& +\eta_{i}(n, t)
\end{aligned}
$$

where $\alpha_{k}$ is referred to as the complex amplitude proportional to the radar cross section (RCS) of the $k^{\text {th }}$ target, and $\eta_{i}(n, t)$ is the zero-mean additive white Gaussian noise at $i^{\text {th }}$ radar node, whose variance is $\sigma^{2}$.

The received signal at $i^{\text {th }}$ radar node, i.e., $y_{i}(n, t)$, is downconverted into the baseband through being multiplied by $e^{-j 2 \pi\left(f_{c}+\mathcal{S}(n) \triangle f\right)\left(t-n T_{c}\right)}$, and is then cross-correlated with the complex envelope of the monotone narrowband pulse transmitted by the same node at time $t \in\left[n T_{c},(n+1) T_{c}\right]$, i.e., $s_{i}\left(t-n T_{c}\right)$, yielding (4), shown at the top of the next page, in which $\tilde{\eta}_{i}(n)=\frac{1}{T_{c}} \int_{n T_{c}}^{(n+1) T_{c}} \eta_{i}(t, n) s_{i}^{*}\left(t-n T_{c}\right) d t$. Due to the near orthogonality of the transmitted waveforms across the radar nodes, the term $A_{2}$ in (4) is negligible compared to the

\footnotetext{
${ }^{1}$ It should be noted that the radar nodes are assumed to use the same carrier frequency for notational brevity. However, in general, there may be some offset between the carrier frequencies of radar nodes, which does not affect the validity of the ensuing derivations and system model.
} 


$$
\begin{aligned}
& z_{i}(n)=\frac{1}{T_{c}} \int_{n T_{c}}^{(n+1) T_{c}} \tilde{y}_{i}(n, t) s_{i}^{*}\left(t-n T_{c}\right) d t=\overbrace{\sum_{k=1}^{K} \frac{\alpha_{k}}{T_{c}} \exp \left\{-j 4 \pi\left(f_{c}+\mathcal{S}(n) \triangle f\right) \tau_{k, i}\right\} \int_{n T_{c}}^{(n+1) T_{c}} s_{i}\left(t-n T_{c}-2 \tau_{k, i}\right) s_{i}^{*}\left(t-n T_{c}\right) d t}^{A_{1}} \\
& +\overbrace{\sum_{k=1}^{K} \sum_{m \neq i} \frac{\alpha_{k}}{T_{c}} \exp \left\{-j 2 \pi\left(f_{c}+\mathcal{S}(n) \triangle f\right)\left(\tau_{k, m}+\tau_{k, i}\right)\right\} \int_{n T_{c}}^{(n+1) T_{c}} s_{m}\left(t-n T_{c}-\tau_{k, m}-\tau_{k, i}\right) s_{i}^{*}\left(t-n T_{c}\right) d t+\tilde{\eta}_{i}(n) .}^{A_{2}}
\end{aligned}
$$

term $A_{1}$. Therefore, (4) is simplified to

$$
z_{i}(n)=\sum_{k=1}^{K} \beta_{k, i} \exp \left\{-j 2 \pi\left(\frac{2 r_{k, i} \triangle f}{c} \mathcal{S}(n)\right)\right\}+\tilde{\eta}_{i}(n)
$$

where $\beta_{k, i}=\alpha_{k} a\left(\frac{2 r_{k, i}}{c}\right) \exp \left\{-j 2 \pi\left(\frac{2 r_{k, i}}{\lambda_{c}}\right)\right\}, a(\tau)=$ $\frac{1}{T_{c}} \int_{n T_{c}}^{(n+1) T_{c}} s_{i}\left(t-n T_{c}-\tau\right) s_{i}^{*}\left(t-n T_{c}\right) d t$, and $\lambda_{c}$ denotes the wavelength of the transmitted signal.

\section{Proposed Localization Algorithm}

In this section, we aim to determine the locations of the targets with respect to the reference point. We propose a twostage decentralized localization algorithm. In the first step, the target detection and precise range estimation are separately done in each radar node. Then, the range measurements are transmitted to a FC for DoA estimation and subsequent localization.

\section{A. Range Estimation}

Let us discretize the range plane on a fine grid so that

$$
2 r_{k, i}=l \vartheta, \quad 0 \leq l \leq L-1
$$

where $\vartheta$ and $\triangle f$ are chosen so that $\frac{\vartheta \triangle f}{c}=\frac{1}{L}$ with $L \gg N$. We can then rewrite (5) as

$$
z_{i}(n)=\sum_{l=0}^{L-1} x_{l} \exp \{-j 2 \pi l \mathcal{S}(n) / L\}+\tilde{\eta}_{i}(n)
$$

where $x_{l}=\alpha_{k} a(l \vartheta / c) e^{-j 2 \pi l \vartheta \triangle f / c}$ if a target is present at the range $l \vartheta$ and zero otherwise. Stacking $z_{i}(n)$ 's into the vector $\mathbf{z}_{i}=\left[z_{1}(0), \cdots, z_{1}(N-1)\right]^{T}$, we can express (7) in the matrix form as

$$
\mathbf{z}_{i}=\Psi_{\mathbf{x}_{i}}+\tilde{\boldsymbol{\eta}}_{i}
$$

where $\mathbf{x}_{i}=\left[x_{0}, \cdots, x_{L-1}\right]^{T}$ and $\boldsymbol{\Psi}$ is a Vandermonde matrix with $[\boldsymbol{\Phi}]_{n, l}=\exp \{-j 2 \pi l \mathcal{S}(n) / N\}$, i.e., it is composed of $N$ rows of the $L \times L$ Discrete Fourier Transform (DFT) matrix. The estimate of target ranges can be found by solving the sparse problem (8) and non-zero index $l$ denotes a target with range $l \vartheta$. In principle, the sparse vector $\mathbf{x}$ can be recovered by solving the $\ell_{1}$-norm optimization problem

$$
\hat{\mathbf{x}}_{i}=\min \left\|\mathbf{x}_{i}\right\|_{1} \quad \text { s.t. } \quad \| \mathbf{z}_{i}-\Psi_{\mathbf{x}_{i} \|_{2} \leq \varepsilon,}
$$

which is also referred to as the Least Absolute Shrinkage and Selection Operator (LASSO) [14]. The LASSO is a convex problem and converges to a global solution with a high probability in polynomial time if the matrix $\Psi$ satisfies the Restricted Isometry Property (RIP) of order $2 K$ with the $2 K$ Restricted Isometry Constant (RIC) $\delta_{2 K}<\frac{2}{3+\sqrt{7 / 4}}$ [15]. The $K$-RIC of a matrix A, i.e., $\delta_{K}$, is the smallest number such that the inequality

$$
\left(1-\delta_{K}\right)\|\mathbf{x}\|_{2}^{2} \leq\|\mathbf{A} \mathbf{x}\|_{2}^{2} \leq\left(1+\delta_{K}\right)\|\mathbf{x}\|_{2}^{2},
$$

holds for all $K$-sparse vetoers $\mathbf{x}$ and a matrix $\mathbf{A}$ fulfills the RIP of order $K$ if $\delta_{K}<1$ [15]. Hence, in order to the range recovery is guaranteed, the subset $\mathcal{S}$ should be chosen such that the RIP of order $2 K$ is satisfied. As mentioned in Section II, the subset $\mathcal{S}$ can be chosen from the set $\mathcal{P}$ in a deterministic or random way. In current work, we select the subset $\mathcal{S}$ uniformly at random form thee set $\mathcal{S}$. In this case, it has been shown in [16] that the matrix $\boldsymbol{\Psi}$ obeys the RIP of order $2 K$ with a high probability if $N \geq C K \log (L / K)$, where $C$ is some constant. Deterministic selection of the subset $\mathcal{S}$ is the topic of the next work, for which the sparest subset of $\mathcal{P}$ should be acquired such that the RIP of order $2 K$ is met.

\section{B. DoA Estimation}

After the distributed sparse processing, the recovered ranges are transmitted to a FC for DoA estimation. According to the law of cosine, we have

$$
r_{k, i}=\sqrt{r_{k}^{2}+d_{i}^{2} \pm 2 r_{k} d_{i} \sin \theta_{k}}
$$

where + and - hold for the nodes on the left and right hand side of the reference point, respectively. Considering the far-field assumption, i.e. $r_{k} \gg d_{m}$, and the Taylor series expansion, (11) can be approximated by

$$
r_{k, i} \simeq r_{k} \pm d_{i} \sin \theta_{k}
$$

Thus, if $\hat{r}_{k, i}$ denotes the recovered range of the $k$ th target with respect to the $i^{\text {th }}$ node and $\hat{r}_{k}$ denote the recovered range of the same target with respect to the reference point, we can argue that,

$$
q_{k, i}=\hat{r}_{k, i}-\hat{r}_{k}= \pm d_{i} \sin \theta_{k}+e_{i},
$$

where $e_{i}$ is the error proceeding from range discretization as well as estimation error which can be generally modeled as a Gaussian random variable with variance $\rho_{i}^{2}$. Staking all $q_{k, i}$ 's 
from different radar nodes for a particular target $k$ into a vector $\mathbf{q}_{k}=\left[q_{k, 1}, \cdots, q_{k, M-1}\right]$, we have

$$
\mathbf{q}_{k}=\mathbf{w} \sin \theta_{k}+\mathbf{e}
$$

where $\mathbf{e}=\left[e_{1}, \cdots, e_{M-1}\right]^{T}$ and $\mathbf{w}=\left[w_{1}, \cdots, w_{M-1}\right]$ with $w_{i}=d_{i}$ if the $i^{\text {th }}$ radar node is on the left hand side of the reference point and $w_{i}=-d_{i}$ otherwise. We can then recast the MLE of $\gamma_{k} \doteq \sin \theta_{k}$ as the following optimization problem,

$$
\begin{array}{cc}
\underset{\gamma_{\mathrm{k}}}{\operatorname{argmin}} & \left(\mathbf{q}_{k}-\mathbf{w} \gamma_{k}\right)^{T} \boldsymbol{\Sigma}^{-1}\left(\mathbf{q}_{k}-\mathbf{w} \gamma_{k}\right), \\
\text { s.t. } & -1 \leq \gamma_{k} \leq 1,
\end{array}
$$

where $\boldsymbol{\Sigma}=\mathrm{E}\left\{\mathbf{e e}^{H}\right\}=\operatorname{diag}\left\{\rho_{1}^{2}, \cdots, \rho_{M-1}^{2}\right\}$. The above constrained optimization problem is convex. Therefore, applying the Karush-Kuhn-Tucker (KKT) optimality conditions [18] leads us to the following estimate of $\nu_{k}$

$$
\hat{\nu}_{k}=\left\{\begin{array}{cc}
\frac{\mathbf{w}^{T} \boldsymbol{\Sigma}^{-1} \mathbf{q}}{\mathbf{w}^{T} \boldsymbol{\Sigma}^{-1} \mathbf{w}} & -1 \leq \frac{\mathbf{w}^{T} \boldsymbol{\Sigma}^{-1} \mathbf{q}}{\mathbf{w}^{T} \boldsymbol{\Sigma}^{-1} \mathbf{w}} \leq 1 \\
1 & \frac{\mathbf{w}^{T} \boldsymbol{\Sigma}^{-1} \mathbf{q}}{\mathbf{w}^{T} \boldsymbol{\Sigma}^{-1} \mathbf{w}}>1 \\
-1 & \frac{\mathbf{w}^{T} \boldsymbol{\Sigma}^{-1} \mathbf{q}}{\mathbf{w}^{T} \boldsymbol{\Sigma}^{-1} \mathbf{w}}<-1
\end{array} .\right.
$$

Taking account of the invariance property of the MLE [17], the estimate of $\theta_{k}$ is obtained as $\hat{\theta}_{k}=\arcsin \hat{\nu}_{k}$. Therefore, the location of the $k^{\text {th }}$ in the polar coordinate system with respect to the reference point is obtained as $\left(\hat{r}_{k}, \hat{\theta}\right)$.

\section{Simulation Results}

In this section, we present numerical results to illustrate the main contributions of the paper which relate to the enhanced performance of proposed localization algorithm arising from sparse sensing.

a) Set-up: We consider an automotive scenario with 5 radar nodes, separated by a distance of $0.1 \mathrm{~m}$, and each containing 4 phased array elements. The phased array in each node creates a wide beam with a width of $\pi / 3$. We consider multiple point targets in the coverage of the beams ensuring that the overlap requirement mentioned in Section II is satisfied. The carrier frequency and the frequency step size are $f_{c}=79 \mathrm{GHz}$ and $\triangle f=10 \mathrm{MHz}$, respectively. Further, we consider a challenging situation with the SNR being $0 \mathrm{~dB}$. The SSFW is generated by selecting a subset $\mathcal{S}$, with $|\mathcal{S}|=\frac{\text { bandwidth }}{\Delta f}$, uniformly at random out of the set $\mathcal{P}=\{1,2, \cdots, 1000\}$. The number of grid points, i.e., $L$, is 1000 . This set up is simulated using the phased-array toolbox of Matlab.

b) Enhanced Range Resolution for given bandwidth: We consider the support recovery error [19] as figure of merit towards illustrating the ability of the proposed scheme in achieving higher range resolution for a given bandwidth. The support recovery error is defined as the error event when at least one target is estimated erroneously [19]. Fig. 2 depicts the support recovery error versus bandwidth for the conventional and proposed technique. 5000 Monte Carlo simulations were performed using independent realization of $K=5$ targets locations, their RCS, the subset $\mathcal{S}$, and noise. It can be seen that the radar system exploiting SSFW and LASSO to recover the target ranges exhibits much better performance than a CSFW radar system using DFT processing [20]. For $1.5 \mathrm{GHz}$ of bandwidth, the proposed technique achieves a $73 \%$ improvement in support recovery error.

c) Multi-target localization: Fig. 3 compares the localization performance of the SSFW and the CSFW. We consider two targets at locations $(8.1 \mathrm{~m}, \pi / 6)$ and $(8.2 \mathrm{~m}, \pi / 4)$ in the polar coordinate system, as shown in Fig. 3. Further, we consider the bandwidth of CSFW to be seven times that of SSFW. It can be seen that the SSFW localizes the two targets properly, while using much lower bandwidth than the CSFW. On the other hand, CSFW estimates the range of one of the targets erroneously and fails to detect the second target, resulting in very poor localization.

\section{CONCLUSION}

The paper considered the problem of multi-target localization using asynchronous MIMO radars with applications to automotive scenario. Arguing the need for high range resolution for accurate localization with affordable bandwidth in such scenarios, the paper considered a new framework based on SSFW and the sparse sensing paradigm. A two stage decentralized procedure for localization is proposed within this framework. The proposed scheme is shown to localize the targets better than the conventional schemes for given bandwidths and offers avenues for further waveform optimization. Enhanced performance and the limited resources consumed, makes the proposed scheme attractive to the industry.

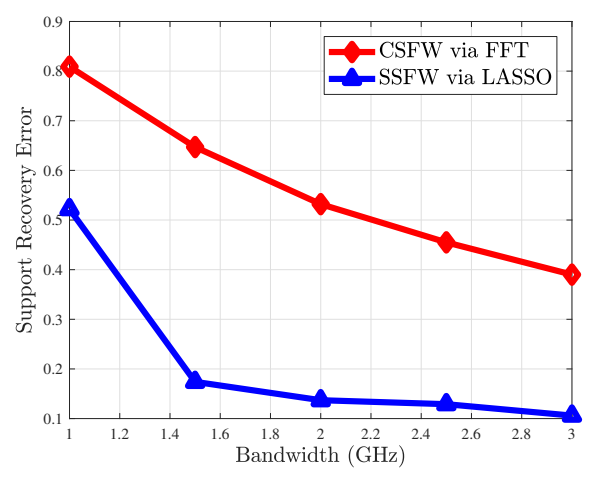

Fig. 2. Support recovery error as a function of the bandwidth.

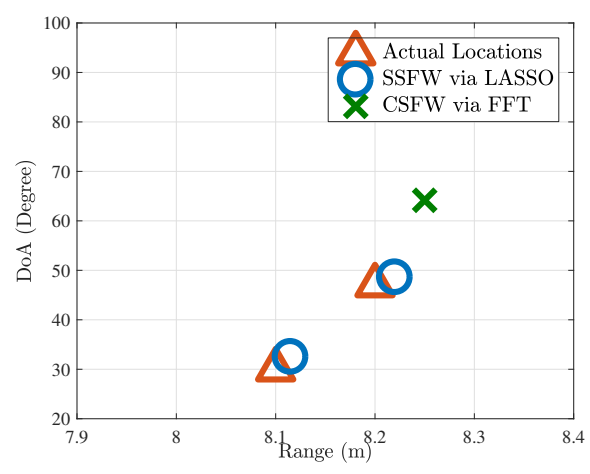

Fig. 3. Localization of two targets. 


\section{REFERENCES}

[1] S. Gogineni, and A. Nehorai. "Target estimation using sparse modeling for distributed MIMO radar," IEEE Trans. Signal Process., vol. 59, no. 11 , pp. 5315-5325, 2011.

[2] Sh. Xu, and K. Dogancay, "Optimal Sensor Placement for 3-D Angleof-Arrival Target Localization," IEEE Trans. Aerosp. Electron. Syst., vol. 53, no. 3, pp. 1196-1211, 2017.

[3] S. Tomic, M. Beko, R. Dinis, and P. Montezuma, "A Closed-Form Solution for RSS/AoA Target Localization by Spherical Coordinates Conversion," IEEE Wireless Commun. Lett., vol. 5, no. 6, pp. 680-683, 2016.

[4] J. Li, and P. Stoica, "MIMO Radar with Colocated Antennas," IEEE Signal Process. Mag., vol. 24, no. 5, pp. 106-114, 2007.

[5] A. Hassanien, M. G. Amin, Y. D. Zhang, and Fauzia Ahmad, "Highresolution single-snapshot DOA estimation in MIMO radar with colocated antennas," in Proc. IEEE Radar Conf. (RadarCon), Arlington, Virginia, pp. 1134-1138, May 2015.

[6] J. Li, and P. Stoica, MIMO Radar Signal Process. John Wiley and Sons Inc., 2009.

[7] F. Folster, H. Rohling, U. Lubbert, "An automotive radar network basaed on $77 \mathrm{GHz}$ FMCW sensors, in Proc. IEEE Radar Conf. (RadarCon), Arlington, Virginia, pp. 871-876, May 2005.

[8] H. Godrich, A. M. Haimovich, and R. S. Blum, "Target Localization Accuracy Gain in MIMO Radar-Based Systems," IEEE Trans. Inf. Theory, vol. 56, no. 6, 2010.

[9] S. Gezici, Z. Tian, G. B. Biannakis, H. Kobayashi, A. F. Molisch, H. V. Poor, and Z. Sahinoglu "Localization via Ultra-Wideband Radios: A Look at Positioning Aspects for Future Sensor Networks," IEEE Signal Process. Mag., vol. 22, no. 4, pp 70-84, 2005.

[10] H. Jamali-Rad, and G. Leus, "Sparsity-Aware Multi-Source TDOA Localization," IEEE Trans. Signal Process., vol. 61, no. 19, pp. 48744887, 2013.

[11] R. Amiri, F. Behnia, and H. Zamani, "Asymptotically Efficient Target Localization From Bistatic Range Measurements in Distributed MIMO Radars," IEEE Signal Process. Let., vol. 24, no. 3, pp. 299-303, 2017.

[12] C.-H. Park, and J.-H. Chang, "Closed-form Localization for Distributed MIMO Radar Systems using Time Delay Measurements," IEEE Trans. Wireless Commun., vol. 16, no. 2, pp. 1480-1490, 2016.

[13] S.P. Chepuri and G. Leus "Sparse sensing for statistical inference," Foundations and Trends in Signal Processing, vol. 9, no. 34, pp 233368, Dec. 2016.

[14] R. Tibshirani, "Regression shrinkage and selection via the lasso," Journal of the Royal Statistic Society, Series B, vol. 58, no. 1, pp 267-288, 1996.

[15] H. Rauhut, "Compressive sensing and structured random matrices." Theoritical Foundations and Numerical Methods for Sparse Recovery, vol. 9, pp. 1-92, 2010.

[16] E. J. Candès, M. B. Wakin, "An Introduction To Compressive Sampling," IEEE Signal Process. Magazine, vol. 25, no. 2, pp. 21-30, March 2008.

[17] S. M. Kay, Fundamentals of Statistical Processing Estimation Theory, Volume I: Estimation Theory. Englewood Cliffs, NJ, USA: Prentice-Hall, 1993.

[18] S. Boyd and L. Vandenberghe, Convex Optimization. Cambridge University Press, 2004.

[19] M. Rossi, A. M. Haimovich, Y. C. Eldar, "Spatial Compressive Sensing for MIMO Radar," IEEE Trans. Signal Process., vol. 62, no. 2, Jan. 2014.

[20] T. Feuillen, A. Mallat, and L. Vandendorpe, "Stepped frequency radar for automotive application: Range-Doppler coupling and distortions analysis," in Proc. IEEE Military Commun. Conf. (MILCOM), Baltimore, Maryland, Nov. 2016. 